\title{
UCRL ${ }^{2} 055-50970483-21$
}

UCRL- -95609

DE87 010876

\section{SUPPRESSION OF THE URANIUM-HYDROGEN REACTION USING HIGH-DOSE CARBON IMPLANTATION}

\author{
R. G. Musket
}
-This paper was prepared for submittal to
The MRS 1987 Spring Meeting
Anaheim, California
$\therefore \quad$ April.21-23, 1987

April 17, 1987

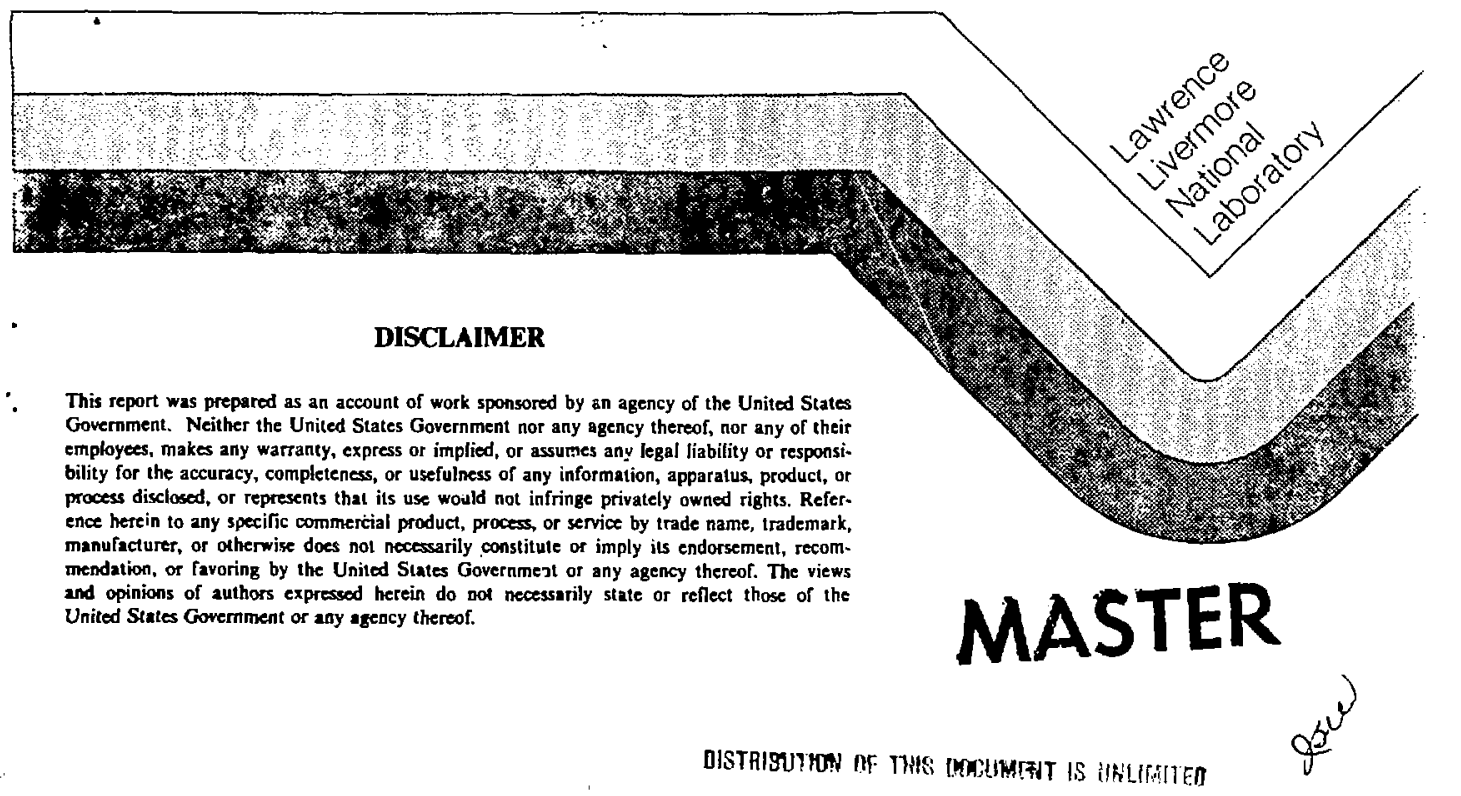


SUPPRESSION OF THE URANIUM-HYDROGEN REACTION USING HIGH-DOSE CARBON IMPLANTATION

R. G. Musket

This paper was prepared for submittal to The MRS 1987 Spring Meeting Anaheim, California April 21-23, 1987

April 17, 1987 


\section{SUPPRESSION OF THE URANIUM-HYOROGEN REACTION \\ USING HIGH-DOSE CARBON IMPLANTATION}

Ronald G. Musket

Lawrence Livermore National Laboratory, P.0. Box 808, Livermore, CA 94550

$\rightarrow$

ABSTRACT

We have previously reported the delay and reduction of the hydriding of uranium by imlantation of oxygen. The reduced hydriding was attributed to the presence of the uranium oxide layer created near room temperature. In this paper we present results for the layers formed by implantation of 80 keV $C^{+}$to a dose of $\mathrm{AE} 17 \mathrm{CLCm^{2 }}$. The carbide layers formed were

charactertzed by nuger electron spectróscopy. Rüfferforobackscattering, andglancing angle $x$-ray diffraction. Hydriding properties of both nonimplanted and implanted uranium were measured for 76 Torr hydrogen at $130^{\circ} \mathrm{C}$. The inplanted specimens had significantly longer incubation tines for the start of the reaction after exposure to hydrogen and less area participating in the reaction.

\section{INTRODUCTION}

We have previousiy shown that ion implantation can markedly affect the hydrogenation of uranium [1]. In this earlier study we compared the hydriding characteristics of the implanted specimens with those of nonimplanted specimens. In particular implantation of large doses (i.e. atoms $/ \mathrm{cm}^{2}$ ) of $0 \frac{5}{2}$ at $180 \mathrm{keV}$ into uranium resulted in the creation of layers that were responsible for (a) increasing the incubation times for the-

- initiation of the reaction after exposure to hydrogen and (b) reducing the - area participating in the reaction. Probable explanations for the reduced hydriding included formation of a uniform, hydride-resistant oxide layer at the higher doses, reduction of the solubility of hydrogen in uranium at the the lower doses, and ion-induced strain in the surface.

We have now implanted carbon ions: in an attempt to create a uniform carbide layer inside the naturally occuring uranium oxide layer. The dose chosen was sufficient to synthesize a:244 $\mathrm{nm}$ thick layer of UC or a $154 \mathrm{~nm}$ layer of $U C_{2}$. Both $U C$ and $U C_{2}$ are thernodynamically stable with respect to $\mathrm{UH}_{3}$ and should resist hydrogenation. In fact, one method of

preparation of uranium carbides is to react fine $\mathrm{UH}_{3}$ powder with fine graphite powder at elevated temperatures (e.g. $>900^{\circ} \mathrm{C}$ ). Ion implantation offers the opportunity to form hydride-resistant uranium carbide layers near the surface of uranium at low temperatures while preserving the bulk properties.

In this paper we present results on the nature of the layers created by low-temperature $\left(<50^{\circ} \mathrm{C}\right.$ ) implantation of $8 E 17 \mathrm{C} / \mathrm{cm}^{2}$ using $80 \mathrm{keV} \mathrm{C}$ and on the comparison of the hydriding characteristics for implanted and non-implanted uraniun specimens. The layers have been characterized using Auger electron spectroscopy (AES). Rutherford backscattering (RBS), and glancing angle $X$-ray diffraction (GXRD).

\section{EXPERIMENTAL CONSIDERATIONS}

The specimens used all came from the same piece of high-purity, depleted uranium used in our previous study [1]. Spectrochenical analys is revealed the following impurities or detection limits (in ppm by weight): 

lapping and mechanical polishing (final step with 1 $\mu \mathrm{m}$ diamond paste) procedures were used to prepare surfaces for electropolishing. The polished specimens were stored in kerosene and/or vacuum until just prior to shipment in all-metal containers to the implanters. The final surface treatment imediately before shipment was an electropolish in a solution of $450 \mathrm{ml}$ ethanol (95\%), $275 \mathrm{ml}$ ethylene glycol, and 275 phosphoric acid (85\%) at room temperature at $13 \vee$ for about three seconds. Finally the specimens were rinsed in de-ionized water and dried in flowing argon gas. These preparation procedures were-applied to both the control and the to-beimplanted specimens. The control specimens were shipped with the finplanted specimens to Insure they experienced the same environmental exposures.

Carbon ions $\left(\mathrm{C}^{+}\right)$were implanted, using an electronically rastered beam. with energies of $80 \mathrm{keV}$ to a dose of $8 E 17 \mathrm{c} / \mathrm{cm}^{2}$ over the central $25-\mathrm{mm}$-diameter area of each specimen. The range and standard deviation for $80 \mathrm{keV} \mathrm{C}^{+}$tons in pure uranfum were calculated using the TRIM program [2] to be 88 and $45 \mathrm{~nm}$, respectively. The maximum temperature during fmplantation was estimated to be $50^{\circ} \mathrm{C}$. After implantation the fmplanted and control specinens were stored under vacuum except when (a) they were renoved for mounting in the hydriding chamber or in an analysis chamber for:AES or RBS and (b) GXRD measurements were performed with the specimens at atmosphere for several hours.

Simulaneous RBS and particle induced $X$-ray errission (PIXE) measurements [3] using 2 Mev helfum lons were combined to determine the depth distribution of the uranium and identify the elements causing the changes from the RBS spectrum for pure uranium. Depth profiles of carbon, oxygen, and uranium were obtained using argon ion sputtering and $5 \mathrm{keV}$ electrons for the AES analysis. GXRO measurements were made using $C u(K) X$-rays at angles of 5-10 degrees from the surface. Standard scanning electron microscopy (SEM) was used to determine the surface microtopography of polished only. implanted, and implanted and hydrided specimens.

The hydriding studies were performed using a vacuum chamber for edgeless studies of gas-metal reactions [4]. In essence, the chamber consisted of two separate vacuum regions with most of the front (i.e. implanted) surface of the specimen exposed to the hydrogen while the other surfaces rena ined under vacuum (< $1 E-5$ Torr). For the present experiments two significant improvements were made to the original chamber design [4]: (a) the specimen was mounted in a vertical plane to ensure the release of loose reaction products and unimpeded access of the gas to the exposed surface and (b) a low-power ( $60 X)$ optical microscope on a XYZ stage was combined with a light source to permit improved observations of the exposed.surface through the sapphire window.

The extent of the reaction was determined by measuring the quantity of hydrogen taken up by the exposed area $\left(3.0 \mathrm{~cm}^{2}\right)$ under isobaric (76 Torr) and isothermal $\left(730^{\circ} \mathrm{C}\right)$ conditions. The relatively short induction times for the non-implanted specimens that were given a 600-grit polish immediately prior to mounting in the hydriding chamber attest to the cleanliness of the hydrogen over the reacting surface. The reaction was stopped by simply evacuating the hydrogen and removing the heating power.

The reaction conditions, which were the same as previously used [1,4], were chosen to fulfill several criteria. A relatively slow reaction rate was desired to permit observation of umambiguous distinctions for the various implantation conditions: however." the rate needed to be sufficient to ensure fairly prompt reaction with the 600-grit polished specimens. The... temperature of $130^{\circ} \mathrm{C}$ meets the above conditions and, in addition, provides -. a reaction that follows a linear law [5] and a hydride attack of nonimplanted uranium that was typical of lower temperatures (i.e. formation of funnel-like hydride pits which penetrate into the reacting sample) [6]. 
For our reaction conditions, the linear reaction rate was estimated from published data [7] to be approxinately $0.8 \mathrm{~mm}$ of pure uranium per minute. The reaction was considered initiated when the amount of hydrogen consumed clearly exceeded 0.017 Torr-liters. For uniform uptake and reaction, 0.017 : Torr-liters of hydrogen would consume about 0.03 mm of pure uraniun (i.e. $1.6 \mathrm{mn} /$ Torr-1iter).

\section{RESULTS AND DISCUSSION}

\section{Characterization of Implanted Layers:}

GXRD measurements indicated that alpha-uranium and $U C_{2}$ were the major phases present in the non-pitted region of an implanted specinen after the hydrogen exposure. $\mathrm{UD}_{2}$ was detected as a trace phase.

AES sputter profiles were measured to determine the depth distributions of carbon, oxygen, and urantum. Figure 1 shows the profile of an implanted specinen after exposure to hydrogen. Hithin the high-carbon region. the carbon Auger peak had the sharp and symetrical shape typical of carbon in carbides. Fron the similarity of this carbon peak structure to that measured for a known UC crystal and that given for UC by Dilliard et al. [8], we concluded that a uranium carbide layer had been formed. The composition of the uniform region of the implanted layer below the surface oxide was taken to be $U C_{2}$ because that was the only carbide phase found by GXRD. Since the sensitivity factor for the uranium OVV peak near 72 eV was taken to be that measured as 0.86 while sputtering the UC crystal, the sensitivity factor for the carbon KLL peak near $270 \mathrm{eV}$ was set at 0.18 for a 11 tines after the first six minutes. The dashed lines are indicative of

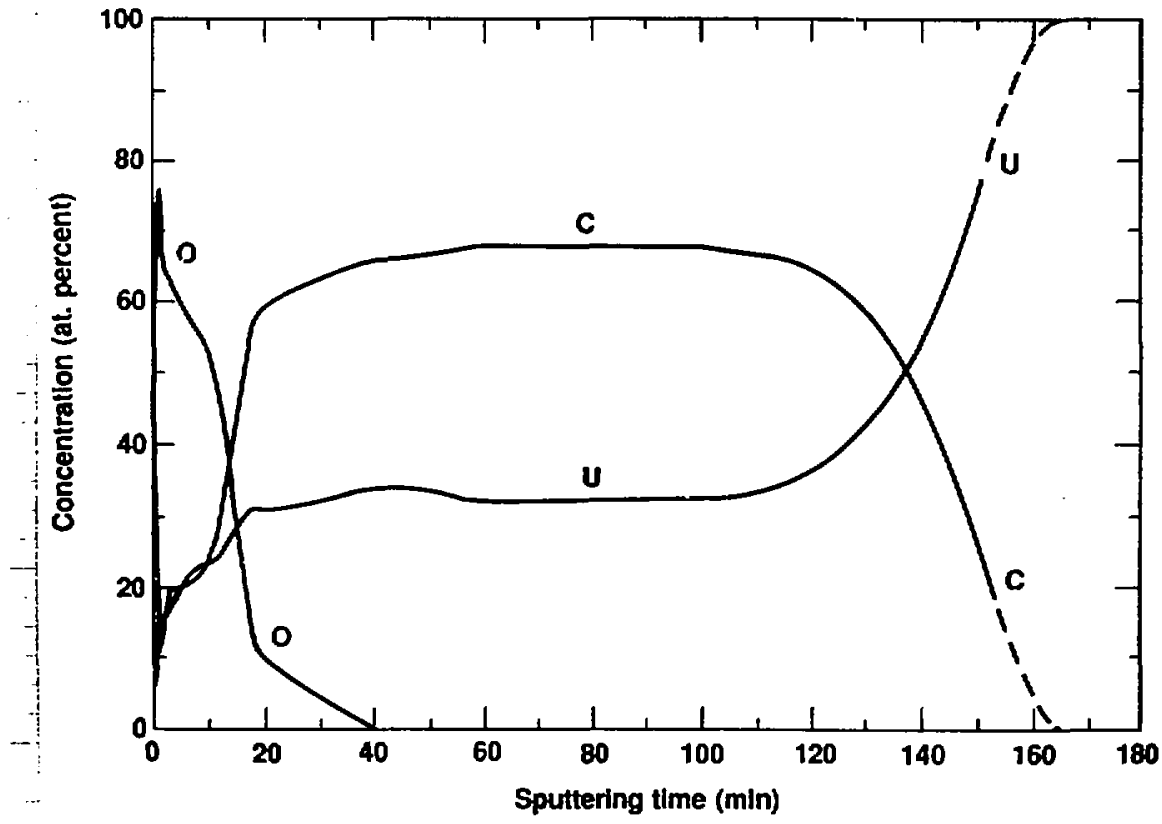

Fig. 1: Auger sputter profile of carbon-inplanted uranium after exposure to hydrogen at 76 Torr and $130^{\circ} \mathrm{C}$. Data taken from a non-pitted region (i.e. center of light spot of Figure 3 ). 
first six minutes of the profile, the carbon peak was clearly that of graphitic cabon, and we used the sensitivity factor measured using a polished graphite standard. The sensitivity factor for oxygen was measured to be 0.36 using an $\mathrm{Al}_{2} \mathrm{O}_{3}$ layer on aluminum.

For an as-implanted specimen. the average $\mathrm{C} / \mathrm{U}$ ratio in the implanted layer was in very good agreement (within two percent) with that for the hydrogen-exposed specimen, but the time to sputter through the layer (i.e. the full width at half maximum or FWHM) was about 30 minutes less than the 128 minutes shown in Figure 1 . This apparent discrepancy may have resultad from slightly different sputtering conditions or real differences in the nature of the carbide layer caused by heating to $130^{\circ} \mathrm{C}$ for two day: and/or exposure to hydrogen. For both specimens, the oxygen content in the implanted layer was below the detection limit of about one atomic percent.

Simultaneous RBS and PIXE measurements showed that carbon, oxysen, and uranium were the only $Z>5$ elements present in the as-implanted specimens. The RBS spectra was dominated by the scattering events from uranium and the distribution of the other elements was inferred from the PIXE. AES, and GXRD results and the change in the uranium scattering height from pure uranium to that in the implanted condition. There was evidence for a surface layer (i.e. oxide) and an interior layer (i.e. implanted layer). Since essentially the same RBS spectrum was found for both the as-implanted: and the implanted and hydrogen-exposed specimens, the $23 \%$ difference in the FWHM of the implanted layers by AES sputter profiling was apparentiy due to variations of the profiling conditions: however the possibility of differentlayer densities cannot be excluded.

Assuming only carbon and uranium were present in the interior layer. the ratio of the shallow minimum in the back-scattering amplitude from uranium in the implanted layer relative to that in pure uranium at the same detected energy was used to calculate the maximum $C / U$ ratio following standard RBS calculationa] procedures [9] coupled with Bragg's rule of linear additivity of atomic stopping cross sections. The pure elementa 7 stopping cross sections were obtained from the program of Zeigler et a]. [10]. Since deviations from Bragg's rule are known to occur for compounds, particularly those containing low-Z elements [11], we used the UC crystal to determine a correction factor (.62) to the stopping cross section for carbon that produced a C/U ratio of 1.00 . A7though this correction factor may not strictly apply for all C/U ratios, we expect that it is better than no correction factor. With this limitation, the peak $c / k$ ratio was found to be 2.57 for two different specimens. The corresponding atomic percents would be 72 for $C$ and $2 B$ for $U$. Since this result applies to the minimum in the uranium scattering signal, it corresponds to the maximum in the $C / U$ :ratio. Consequentily, the average Tayer composition would be in reasonable :agreement with the atomic percents for $U C_{2}$ : 67 for $C$ and 33 for $U$. However,-the apparent carbon richness may be explained by a region of the layer containing some implanted carbon in excess of that required to form $\mathrm{UC}_{2}$.

The thickness of the implanted layers was determined from the RBS spectra by considering the energy difference between ijelium scattered from uranium near the oxide/carbide and the carbide/uranium interfaces. Essentially the same thickness was found for the as-implanted and the implanted and hydrogen-exposed specimens. The calculation was performed assuming two different $C / U$ ratios: 2.00 and 2.57 . For $C / U=2.00$ the layer thickness was about $4.2 E 17 U C_{2} / \mathrm{cm}^{2}$; for $C / U=2.57$ the thickness

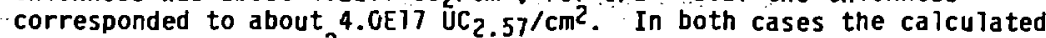
quantity of carbon $/ \mathrm{cm}^{2}$ (i.e. $8.5 E Y 7$ and $10.3 E 77$. respectively) was in reasonable agreement with the implanted dose of $8 \mathrm{E} 17 \mathrm{c} / \mathrm{cm}^{2}$. 
Hydriding Experiments:

The hydrogen consumption results are displayed in Figure 2. Times for the initiation of the reaction were $<1$ and 23 minutes for the 600-grit polished and electropolished control specimens, respectively. These $t$ imes

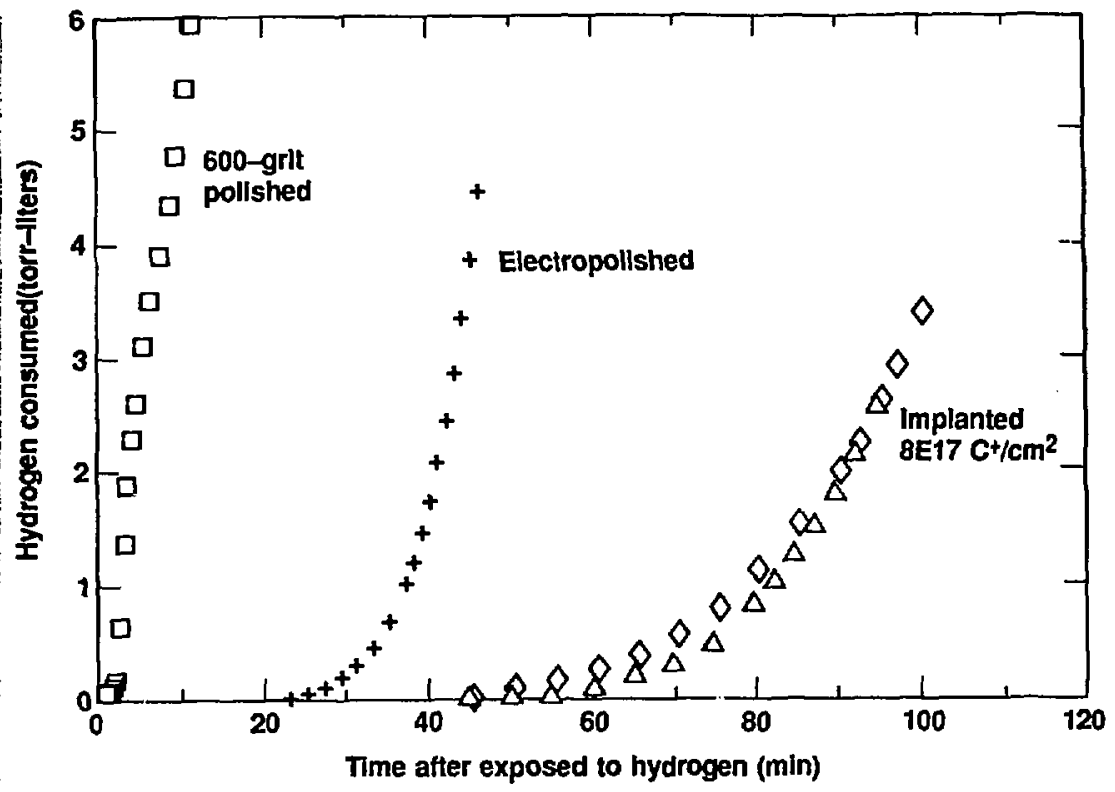

Fig.2: Comparison of the reaction of 76 Torr of hydrogen at $130^{\circ} \mathrm{C}$ with two implanted and two ron-implanted uranium specimens.

are consistent with those measured at other times for similar specimens. Induction times for the specimens implanted with carbon were both about 45 min. This increase in the induction time is significant and clearly shows the effect of the implanted layer. The reaction rates at the end of the exposures for the 600-grit pulished and electropolished uranium specimens both corresponded to consumption of about 0.86 un of uranium per minute. which agrees with that expected from the literature [7]. In contrast, the final rates for the two implanted specimens were both approximately $0.23 \mathrm{w} \mathrm{U} / \mathrm{min}$. The apparently lower reaction rate of the implanted - specimens is a consequence of the smaller fraction of the surface

$\therefore$ participating in the reaction allowing for the internal surface areas of the pits (Figure 3). The AES profile data of Figure 1 was obtained from the - center of the bright, sputtered spot of Figure 3.

$-i \quad$ In agreement with the morphology attendent with the oxygen-implanted and hydrogen-exposed surfaces [1], the surfaces of the carbon-implanted, hydrogen-exposed specinens, as revealed by SEM at lokX, had large, nonpitted areas very similar to the smooth. pre-exposured surface. Thus, the implanted layer greatly suppressed the uranium-hydrogen reaction over most of the exposed surface area. Identification of the mechanisms responsible f for this suppression has not been unanbigously made, but the potential candidates are similar to those proposed for the oxygen implantations [1]: (a) a uniform, hydride-resistant carbide layer, (b) reduction of the solubility of hydrogen in uranium below the carbide layer where the carbon 


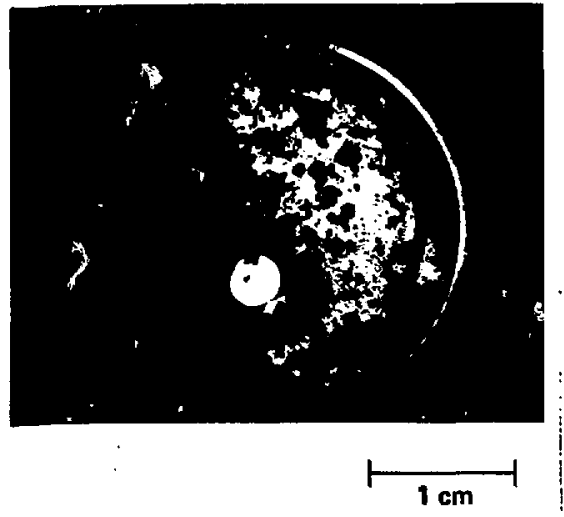

Fig.3: Optical micrograph of carbonimplanted uranium after exposure to hydrogen at 76 Torr and $130^{\circ} \mathrm{C}$ for 95 minutes. concentration is lower, and (c) ion-induced strain in the surface. Experiments are in progress to evaluate the importance of ioninduced strain on tise hydrogenation of uranium.

The occurance of hydriding at isolated spots is also similar to that previously observed for the oxygen-implanted specimens [1]. Presumably the hydriding sites correspond to small areas that were not modified the same as the vast majority of the surface. Such small areas could have resulted from (a) implantation into impurity inclusions and/or (b) lack of implantation in suriaces inaccessible to the ions (e.g. sides of some defects. or areas masked by dust particles). Some effects of the presence of dust particles are presently being evaluated.

\section{CONCLUDING REMARKS}

Implantation of $80 \mathrm{keV} \mathrm{c}^{+}$into uranium to a fluence of $\left.8 \mathrm{E}\right] 7 \mathrm{c} / \mathrm{cm}^{2}$ near room temperature results in the formation of a uranium carbide layer with a composition nesr that of $U C_{2}$. The presence of this layer modifies the reaction of hydrogen at 76 Torr and $130^{\circ} \mathrm{C}$ with the uranium. Implanted specimens exhibited significantly longer incubation times for the start of the reaction after exposure to hydrogen and considerably smaller areas participating in the reaction. Combining this result with our previous results for oxide layers formed by implantation of oxygen [1], we can conclude, in general, that formation by ion implantation of compound layers that are thermodynamically stable with respect to hydrogen should suppress the uranium-hydrogen reaction. Future studies should address the detailed phenomena responsible for the reduced reaction.

This work and our previous study with oxygen-implanted uranium [1] demonstrated reduced hydriding for ion implanted uranium specimens. Hof fmann et a1. [12] have shown that implantation of palladium enhances the uptake of hydrogen by titanium. Although the these effects of ion implantation have only been reported for uranium and titanium, there are most certainly other other materials whose hydriding properties could be markedly modified by ion implantation.

\section{ACKMOWLEDGEMENTS}

It is a pleasure to acknowledge $C$. A. Colmenares for providing the uranium material; H. C. Hayden of the University of Connecticut for the expert ion implantations; R. G. Patterson for the specimen preparation and hydriding measurements: J. R. Thournir for measuring the AES profiles; $S$. J. Holmes for assistance with the RBS and PIXE analyses; G. S. Smith and W. R. Wade for the $X$-ray diffraction measurements and interpretations; and $D$. $D$. McCoy and J. H. Yoshiyama for the SEM micrographs. Work performed under the auspices of the U.S. Department of Energy by the Lawrence Livermore Nationa? Laboratory under contract No. H-7405-ENG-4B. 
1. R.G. Musket, G. Robinson-weis, and R.G. Patterson, Mat. Res. Soc. Symp. Proc., Vol. 27 (1984), p. 753.

2. J.P. Biersack and L.G. Haggmark, Nucl.Instr. and Methods 174, 257 (1980).

3. R.G. Musket, Nuc1. Instr. and Methods Phys. Res. 218, 420 (1983).

4. R.G. Patterson and R.G. Musket, J. Vac. Sci. Techno1. A2, 82 (1984).

5. W.M. Mueller, J.P. Blackledge, and G.G. Libowitz (editors), Metal Hydrides (Academic Press. NY, 1968).

6. 3. E:och and M.H. Mintz, J. Nuc1. Mater. 110, 251 (1982).

7. J. Bloch and M.H. Mintz, J. Less-Common Met. 81, 301 (1981).

8. J.G. Dillard, H. Moers, H. Klewe-Nebenius, G. Kirch, G. Pfennig, and K.J. Ache, J. Phys. Chem 98, 5345 (1984).

9. W... Chu, J.W. Mayer, and M.A. Kicolet, Backscattering Spectrometry (Academic Press, NY, 197B).

10. J.F. Zjegler, J.P. Biersack, and U. Littmark, The Stopping and Range of Ions in Solids, Vol. 1 (Pergamon Press, NY, 1985).

11. O.I. The:aites, Radiat. Res. 95, 495 (1983).

12. B. Hoffmann, H. Baumann, and F. Rauch, Nucl. Instr. and Methods Phys. Res. B15, 361 (1986). 